\title{
An integrated biopsychosocial model of childhood maltreatment and psychosis
}

\author{
Victoria Barker, Andrew Gumley, Matthias Schwannauer and Stephen M. Lawrie
}

\section{Summary}

There is now a well-established link between childhood maltreatment and psychosis. It is, however, unclear what the mechanisms are by which this occurs. Here, we propose a pathway linking the experience of childhood maltreatment with biological changes in the brain and suggest a psychological intervention to ameliorate its effects.

\section{Declaration of interest}

None.
Victoria Barker is a clinical lecturer in general adult psychiatry in the Centre for Brain Sciences at the University of Edinburgh. Andrew Gumley is a Professor of Psychological Therapy in the Institute of Health and Wellbeing, University of Glasgow. Matthias Schwannauer is a Professor of Clinical Psychology and Head of Clinical and Health Psychology in the Centre for Brain Sciences at the University of Edinburgh. Stephen M. Lawrie is Head of the Division of Psychiatry, Professor of Psychiatry \& Neuroimaging and Director of PSYSTAR in the Centre for Brain Sciences in the University of Edinburgh.

The stress-diathesis model suggests that a biologically driven (genetic) predisposition interacting with environmental factors produces an individual's phenotype. ${ }^{1}$ There is a need, however for a detailed integrated biopsychosocial/psychobiological model that explains how such factors interact to lead on to psychosis. It is well established that childhood maltreatment increases the risk of developing psychosis and in particular hallucinations and delusions, ${ }^{2}$ but also other psychiatric disorders including affective and anxiety disorders. It is unclear what determines an individual's vulnerability to different psychiatric disorders given similar environmental exposures but one likely influence is genetic vulnerability. Here we describe one likely mechanism by which childhood maltreatment may lead on to the development of psychosis years or even decades later.

\section{Childhood maltreatment, the hypothalamic-pituitary-adrenal axis and psychosis}

Hypothalamic-pituitary-adrenal (HPA) axis activation occurs in response to environmental stress and has been implicated in the pathway from chronic stress, such as childhood maltreatment, to a variety of psychiatric disorders. Read et al were among the first to propose a traumagenic neurodevelopmental model of schizophrenia mediated by the HPA axis. ${ }^{3}$ Adults who have experienced childhood maltreatment show hyper-reactivity and persistent sensitisation of the HPA stress response. ${ }^{4}$ The hippocampus is involved in terminating the stress response through glucocorticoid-mediated negative feedback on the HPA axis. Acute and chronic stressors impair activation of this negative feedback system and lead to an increased response to subsequent normal stresses throughout life (for review see Maras \& Baram ${ }^{5}$ ). Numerous studies have reported hippocampal volume alterations in adults who were subject to childhood maltreatment. It has been proposed that $N$-methyl-D-aspartate (NMDA) glutamate receptor antagonism and hypofunction can damage brain areas, including the hippocampus, and also lead to HPA hyperactivation ${ }^{6}$ and increased mesolimbic dopamine.

Individuals with schizophrenia have an altered HPA stress response and HPA axis hyperactivity may mediate the relationship between stress and psychosis. ${ }^{7}$ Stress increases dopamine release, which is in turn associated with greater cortisol response to further challenges (for a relevant review see Howes \& Murray ${ }^{8}$ ). Healthy adults who report low maternal care as children, those who have experienced childhood maltreatment and those with schizophrenia show increased dopamine release to social stressors, mediated by cortisol. Thus, acute and chronic stress leads to HPA axis hyper-reactivity, which may result in reduced hippocampal volume, increased dopamine release and psychosis.

\section{The role of BDNF}

Brain-derived neurotrophic factor (BDNF) is involved in the regulation of the stress response and hippocampal plasticity. Acute and chronic stress reduce BDNF mRNA expression in the hippocampus and impair neuronal branching and neurogenesis. BDNF expression is regulated by NMDA receptor activation and increases with glutamate release. Chronic activation of glucocorticoid receptors in response to stress induces receptor downregulation and suppresses BDNF expression. High maternal care is associated with increased expression of NMDA receptors in the hippocampus resulting in elevated BDNF expression. ${ }^{9}$ There is evidence that BDNF levels are disrupted in schizophrenia. ${ }^{10}$ In patients with first-episode psychosis, childhood maltreatment and number of recent stressful life events have been shown to be negatively correlated with BDNF mRNA levels ${ }^{11}$ and BDNF gene expression and higher cortisol levels were independently associated with smaller left hippocampal volume. Thus, the reduced hippocampal volume in response to childhood maltreatment and HPA axis hyper-reactivity appears to be mediated by reduced BDNF and NMDA expression, all of which are suggested as being involved in the development of psychosis.

\section{The role of oxytocin}

One neuropeptide that has been linked to childhood maltreatment and the HPA axis and has also been implicated in the development of psychosis is oxytocin (for a review see Macdonald \& Feifel ${ }^{12}$ ). Animal studies have shown a direct effect of oxytocin on dopamine release within the mesocorticolimbic system and the strength of this connection is associated with levels of maternal care. ${ }^{13}$ Plasma oxytocin in patients with schizophrenia is positively correlated with 
hippocampal-mediated HPA feedback. ${ }^{14}$ Oxytocin is well known to interact with the HPA axis and under normal circumstances attenuates the HPA axis stress response. Chronic stressor exposure mediated through glucocorticoid receptor activation can downregulate the oxytocin system. ${ }^{15}$ Those who have experienced childhood maltreatment have decreased concentrations of oxytocin in their cerebrospinal fluid. ${ }^{16}$ BDNF upregulates oxytocin secretion in primary neuronal culture suggesting that these molecules may both be involved in mediating the response to childhood maltreatment ${ }^{17}$ and a reduction in BDNF is associated with reduced oxytocin.

\section{Epigenetics, the interface between environmental and genetic risk factors for psychosis}

Epigenetic changes enable external environmental factors to influence long-lasting phenotypic change. Thus, epigenetic processes may represent the interface between environmental and genetic risk factors for psychosis and they have been implicated as mediating the effects of childhood maltreatment in the development of psychosis. ${ }^{18}$ In rat pups, low maternal care leads to increased DNA methylation of the promoter region of the glucocorticoid receptor gene in the hippocampus, which in turn results in reduced numbers of glucocorticoid receptors and HPA hyper-reactivity throughout life. ${ }^{19}$ This can be reversed by crossfostering to a different maternal carer as well as pharmacological manipulation, which raises the prospect that such developmental changes may be amenable to various therapeutic approaches. This same epigenetic process has been found in response to exposure to childhood maltreatment in humans. ${ }^{20}$ Epigenetic variations in the oxytocin system have been suggested as being involved in the mechanisms mediating the long-term influence of early adverse experiences. ${ }^{21}$ DNA methylation also plays a role in BDNF gene regulation and this has been implicated in modulating the effects of childhood maltreatment ${ }^{22}$ and in the development of schizophrenia. The hippocampus and prefrontal cortex of individuals with schizophrenia have been found to have decreased BDNF protein and mRNA levels post-mortem and DNA methylation could be responsible for this. There is therefore evidence to suggest a pathway from childhood maltreatment to psychosis via HPA axis hyperactivation, reduced hippocampal BDNF and oxytocin levels and NMDA hypoactivation by glutamate leading to decreased hippocampal volume and increased mesolimbic dopamine. There is also evidence that elements of this pathway are mediated by epigenetic processes including methylation of the glucocorticoid receptor, BDNF and oxytocin receptor genes (Fig. 1). It is important to acknowledge that much of the work done in elucidating the mechanisms involved in this pathway was done using animal models rather than in humans and it will be imperative that future studies be undertaken examining whether HPA activity, glucocorticoid receptor, BDNF and oxytocin receptor gene methylation status are modified by the experience of childhood maltreatment and ascertaining whether this is associated with structural and functional changes, in particular in the hippocampus.

\section{Towards new treatments}

Once we understand how childhood maltreatment is involved in the development of psychosis we can look at new targeted

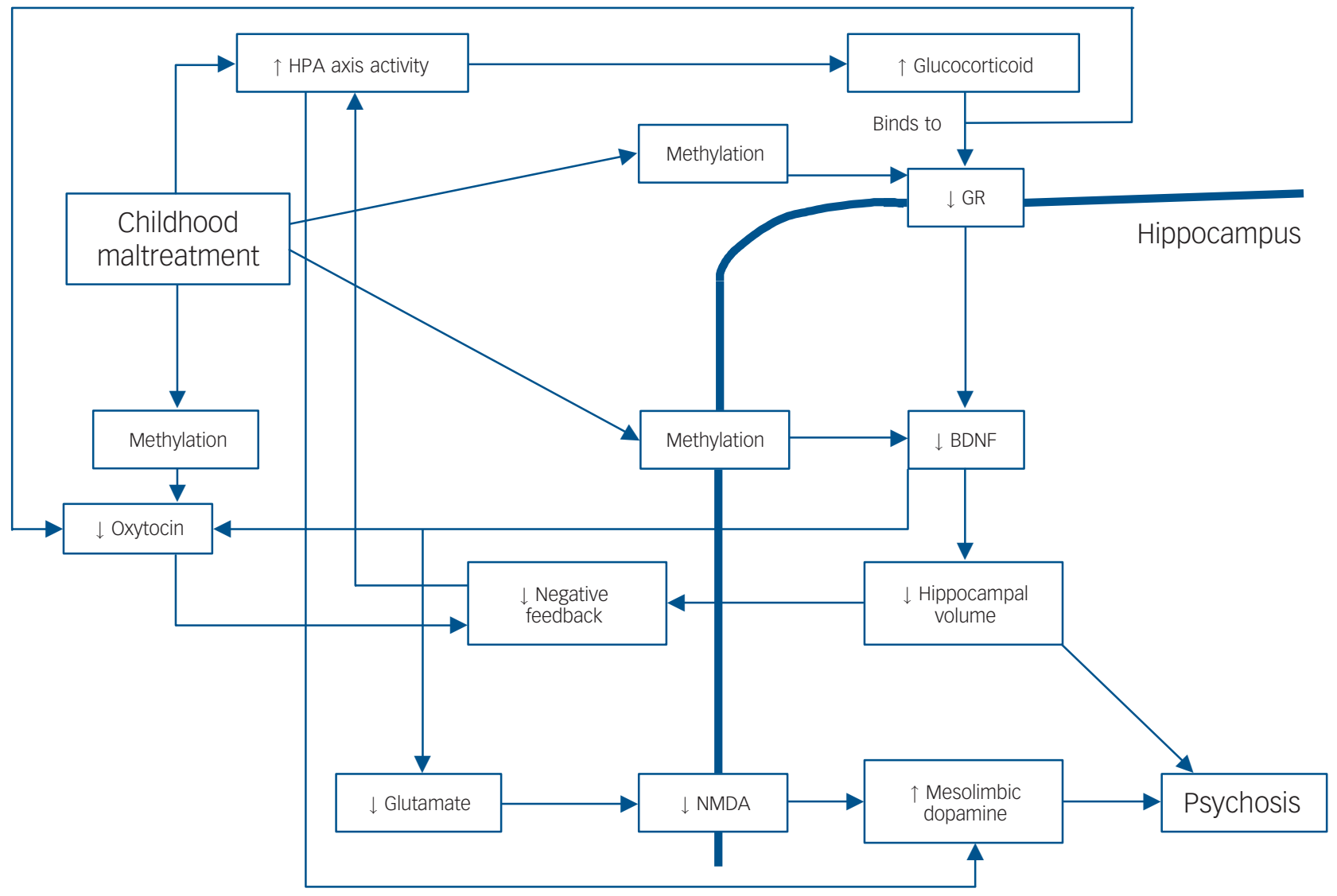

Fig. 1 A pathway from childhood maltreatment to psychosis. 
interventions and examine their impact on this pathway. Attachment theory provides a psychological framework for conceptualising the role of social cognition, interpersonal experience and regulation of affect in the development of both interpersonal functioning and psychological distress. ${ }^{23}$ The greatest threats to the integrity of the attachment system are events and processes that threaten the security of the attachment bond such as extended separation, loss, trauma and neglect. ${ }^{24}$ It is well established that maltreated children are more likely to be insecurely attached and to show a disorganised pattern of attachment. Disorganised attachment stems from a conflict between the attachment system and the flght-flight system. These systems normally operate in union (i.e. flight from the source of fear to find refuge near the attachment figure). In infant-caregiver interactions where the caregiver is at the same time the source of, and the solution to, the infant's fear such as in childhood maltreatment these systems are at odds and disorganised attachment develops (for a review see Cicchetti \& Toth ${ }^{25}$ ). Oxytocin is well established as being associated with attachment and enhances the experience of attachment security. ${ }^{26}$ Disorganised attachment may evolve into an adult dismissing attachment style. Individuals with psychosis more commonly have insecure-avoidant/dismissing attachment styles (for a review see Berry et $a l^{27}$ ). This attachment style has been found to relate to higher levels of positive symptoms and to a poorer therapeutic relationship in people with chronic schizophrenia or schizoaffective disorder and to poorer symptomatic recovery prospectively in individuals with first-episode psychosis. ${ }^{28}$ Attachment can be seen as indicative of early care and it is possible that the development of insecure attachment is associated with the biological factors in Fig. 1. This may serve to increase the liability to psychosis, perhaps precipitated by additional stressors or developmental changes.

There is evidence to suggest that attachment security can change as a result of significant interpersonal experiences including psychological therapy. ${ }^{23}$ So, interventions that seek to establish a new model of secure attachment or a new experience of care could have an impact on the biological pathway described above and reduce psychotic symptoms. Indeed, a recent study found a positive association between childhood maltreatment and BDNF methylation status in individuals with borderline personality disorder, with a decrease in BDNF methylation status in those that responded to intensive dialectical behaviour therapy. ${ }^{29}$

One potential therapy for patients with psychosis and a history of childhood maltreatment is mentalisation-based treatment. Mentalisation concerns the ability of the individual to understand and infer the mental states of both self, and others, and the impact of mental states on one's cognitions, affective state, sense of agency and behaviour. The capacity to mentalise develops within the context of secure attachment. Negative interpersonal experiences during childhood disrupt an individual's development of mentalisation skills. It has been shown that in those with a dismissing attachment style and individuals with psychosis there is impaired mentalisation capacity. ${ }^{30}$ Mentalisation-based treatment aims to re-establish an attachment relationship with the patient and thus ameliorate deficits in mentalisation. It was first developed to treat patients with borderline personality disorder, a group of patients who have been shown to have attachment difficulties, who have commonly experienced childhood maltreatment and a proportion of whom experience psychotic symptoms. It now has an established evidence base for its efficacy in this group (for Cochrane review see Stoffers et $a l^{31}$ ).

An understanding of the biological processes involved in mediating the influence of childhood maltreatment on the development of psychosis and other psychiatric disorders allows us to develop interventions based on a strong theoretical foundation. It is even possible that as these epigenetic changes in response to childhood maltreatment have been proposed to be involved in the pathogenesis of several different psychiatric disorders including depression, bipolar disorder and schizophrenia, there may be a role for a single therapeutic intervention targeted at reversing the biological effects of childhood maltreatment in patients with a wide range of psychiatric disorders.

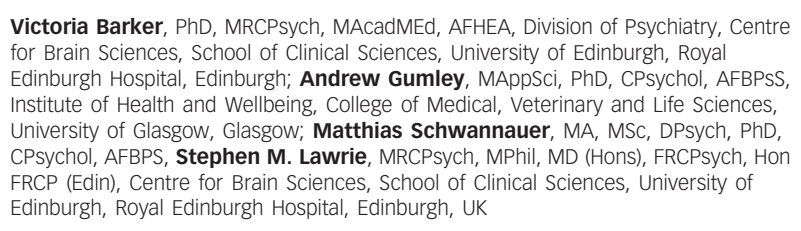

Victoria Barker, PhD, MRCPsych, MAcadMEd, AFHEA, Division of Psychiatry, Centre for Brain Sciences, School of Clinical Sciences, University of Edinburgh, Royal Edinburgh Hospital, Edinburgh; Andrew Gumley, MAppSci, PhD, CPSychol, AFBPSS, Institute of Health and Wellbeing, College of Medical, Veterinary and Life Sciences, University of Glasgow, Glasgow; Matthias Schwannauer, MA, MSC, DPsych, PhD, CPSychol, AFBPS, Stephen M. Lawrie, MRCPSych, MPhil, MD (Hons), FRCPsych, Hon FRCP (Edin), Centre for Brain Sciences, School of Clinical Sciences, University of Edinburgh, Royal Edinburgh Hospital, Edinburgh, UK

Correspondence: Victoria Barker, University of Edinburgh, 48 Duddingston Park, Edinburgh EH15 1JY, UK. Email: victoria.barker@ed.ac.uk

First received 21 Mar 2014, final revision 3 Nov 2014, accepted 6 Nov 2014

\section{References}

1 Zubin J, Spring B. Vulnerability - a new view of schizophrenia. J Abnorm Psychol 1977; 86: 103-26.

2 Varese F, Smeets F, Drukker M, Lieverse R, Lataster T, Viechtbauer W, et al. Childhood adversities increase the risk of psychosis: a meta-analysis of patient-control, prospective- and cross-sectional cohort studies. Schizophr Bull 2012; 38: 661-71.

3 Read J, Perry BD, Moskowitz A, Connolly J. The contribution of early traumatic events to schizophrenia in some patients: a traumagenic neurodevelopmental model. Psychiatry 2001; 64: 319-45.

4 Heim C, Newport DJ, Heit S, Graham YP, Wilcox M, Bonsall R, et al. Pituitary-adrenal and autonomic responses to stress in women after sexual and physical abuse in childhood. JAMA 2000; 284: 592-7.

5 Maras PM, Baram TZ. Sculpting the hippocampus from within: stress, spines, and CRH. Trends Neurosci 2012; 35: 315-24.

6 Olney JW, Farber NB., Glutamate receptor dysfunction and schizophrenia. Arch Gen Psychiatry 1995; 52: 998-1007.

7 Walker E, Mittal V, Tessner K. Stress and the hypothalamic pituitary adrenal axis in the developmental course of schizophrenia. Annu Rev Clin Psychol 2008; 4: 189-216.

8 Howes OD, Murray RM. Schizophrenia: an integrated sociodevelopmentalcognitive model. Lancet 2014; 383: 1677-87.

9 Meaney MJ. Maternal care, gene expression, and the transmission of individual differences in stress reactivity across generations. Annu Rev Neurosci 2001; 24: 1161-92.

10 Shoval G, Weizman A. The possible role of neurotrophins in the pathogenesis and therapy of schizophrenia. Eur Neuropsychopharmacol 2005; 15: 319-29.

11 Mondelli V, Cattaneo A, Belvederi Murri M, Di Forti M, Handley R, Hepgul N, et al. Stress and inflammation reduce brain-derived neurotrophic factor expression in first-episode psychosis: a pathway to smaller hippocampal volume. J Clin Psychiatry 2011; 72: 1677-84

12 Macdonald K, Feifel D. Oxytocin in schizophrenia: a review of evidence for its therapeutic effects. Acta Neuropsychiatr 2012; 24: 130-46.

13 Strathearn L. Maternal neglect: oxytocin, dopamine and the neurobiology of attachment. J Neuroendocrinol 2011; 23: 1054-65.

14 Goldman M, Marlow-O'Connor M, Torres I, Carter CS. Diminished plasma oxytocin in schizophrenic patients with neuroendocrine dysfunction and emotional deficits. Schizophr Res 2008; 98: 247-55.

15 Neumann ID. Involvement of the brain oxytocin system in stress coping: interactions with the hypothalamo-pituitary-adrenal axis. Prog Brain Res 2002; 139: 147-62.

16 Heim C, Young $L$, Newport DJ, Mletzko T, Miller AH, Nemeroff CB. Lower CSF oxytocin concentrations in women with a history of childhood abuse. Mol Psychiatry 2009; 14: 954-8.

17 Moreno G, Piermaria J, Gaillard RC, Spinedi E. In vitro functionality of isolated embryonic hypothalamic vasopressinergic and oxytocinergic neurons: modulatory effects of brain-derived neurotrophic factor and angiotensin II. Endocrine 2011; 39: 83-8.

18 Read J, Bentall RP, Fosse R. Time to abandon the bio-bio-bio model of psychosis: exploring the epigenetic and psychological mechanisms by which 
adverse life events lead to psychotic symptoms. Epidemiol Psichiatr Soc 2009; 18: 299-310.

19 Weaver IC, Cervoni N, Champagne FA, D'Alessio AC, Sharma S, et al. Epigenetic programming by maternal behavior. Nat Neurosci 2004; 7: 847-54.

20 McGowan PO, Sasaki A, D'Alessio AC, Dymov S, Labonté B, Szyf M, et al. Epigenetic regulation of the glucocorticoid receptor in human brain associates with childhood abuse. Nat Neurosci 2009; 12: 342-8.

21 Kumsta R, Hummel E, Chen FS, Heinrichs M. Epigenetic regulation of the oxytocin receptor gene: implications for behavioral neuroscience. Front Neurosci 2013; 7: 83.

22 Roth TL Lubin FD, Funk AJ, Sweatt JD. Lasting epigenetic influence of early-life adversity on the BDNF gene. Biol Psychiatry 2009; 65: 760-9.

23 Mallinckrodt B. Attachment, social competencies, social support and interpersonal processes in psychotherapy. Psychother Res 2000; 10: 239-66.

24 Bowlby J. Attachment and Loss Vol. 2: Separation, Anger and Anxiety. Hogarth Press, 1973

25 Cicchetti D, Toth SL. A developmental psychopathology perspective on child abuse and neglect. J Am Acad Child Adolesc Psychiatry 1995; 34: 541-65.
26 Buchheim A, Heinrichs M, George C, Pokorny D, Koops E, Henningsen P, et al. Oxytocin enhances the experience of attachment security. Psychoneuroendocrinology 2009; 34: 1417-22.

27 Berry K, Barrowclough C, Wearden A. A review of the role of adult attachment style in psychosis: unexplored issues and questions for further research. Clin Psychol Rev 2007; 27: 458-75.

28 Gumley Al, Schwannauer M, Macbeth A, Fisher R, Clark S, Rattrie L, et al. Insight, duration of untreated psychosis and attachment in first-episode psychosis: prospective study of psychiatric recovery over 12-month follow-up. Br J Psychiatry 2014; 205: 60-7.

29 Perroud N, Salzmann A, Prada P, Nicastro R, Hoeppli ME, et al. Response to psychotherapy in borderline personality disorder and methylation status of the BDNF gene. Transl Psychiatry 2013; 3: e207.

30 MacBeth A, Gumley A, Schwannauer M, Fisher R. Attachment states of mind, mentalization, and their correlates in a first-episode psychosis sample. Psychol Psychother 2011; 84: 42-57.

31 Stoffers JM, Völlm BA, Rücker G, Timmer A, Huband N, Lieb K. Psychological therapies for people with borderline personality disorder. Cochrane Database Syst Rev 2012; 8: CD005652.

\section{poems

\section{You're not welcome in here}

\section{Hinesh Topiwala}

I wipe away the tears and dust you down, after you've had a bad day.

I wake you up in the morning, with the promise that things will get better.

So carefully and tenderly,

I give you the handle of the mug of hot milk.

Then I tie your shoe laces into giant bows, together we cheer.

Here we are,

ready to face the day.

What a team we make,

an old head and a young body.

Just me and me. 